\title{
Psychological Trauma and Physical Health: A Psychoneuroimmunology Approach to Etiology of Negative Health Effects and Possible Interventions
}

\author{
Kathleen Kendall-Tackett \\ Texas Tech University School of Medicine
}

\begin{abstract}
People who have experienced traumatic events have higher rates than the general population of a wide range of serious and life-threatening illnesses including cardiovascular disease, diabetes, gastrointestinal disorders, and cancer. An important question, for both researchers and clinicians, is why this occurs. Researchers have discovered that traumatic events dysregulate the hypothalamic-pituitary-adrenal axis and sympathetic nervous system. More recently, research from the field of psychoneuroimmunology (PNI) suggests that traumatic life events can lead to health problems through dysregulation of another key system: the inflammatory response. Prior trauma "primes" the inflammatory response system so that it reacts more rapidly to subsequent life stressors. Elevated inflammation has an etiologic role in many chronic illnesses. Recent PNI studies also suggest some interventions that can serve as adjuncts to traditional trauma treatment. These treatments include long-chain omega-3 fatty acids, exercise, and sleep interventions. Each of these interventions downregulates inflammation, which will likely halt the progression to chronic disease for some trauma survivors.
\end{abstract}

Keywords: trauma, inflammation, depression, PTSD, omega-3s

For more than a decade, researchers have explored the impact of traumatic events on physical health. Trauma survivors often have significant physical health problems that can linger for many years after the traumatic event has ended and are often above and beyond the effects of direct physical injury (KendallTackett, 2007; Kibler, Joshi, \& Hughes, in press). The question of trauma's impact and health is at the junction of mind-body medicine. It has important implications for not only health care costs, but also for trauma practice, and how we can best meet the needs of people who have experienced trauma. In this article, I review research on psychological trauma and physical health using a psychoneuroimmunology (PNI) approach. PNI research can describe some of the possible mechanisms by which trauma affects health. It also suggests some viable additions to traditional trauma treatments that will address the physical health sequelae.

Correspondence concerning this article should be addressed to Kathleen Kendall-Tackett, Department of Pediatrics, Texas Tech University School of Medicine, $1400 \mathrm{~N}$. Coulter Street, Rm 4301, Amarillo, TX 79106. E-mail: kathleen.kendall-tackett@ttuhsc.edu

\section{Trauma Increases the Risk of Health Problems}

The Adverse Childhood Experiences (ACE) study, with more than 17,000 participants from a large California health maintenance organization (HMO), was the first large-scale study to demonstrate the link between childhood trauma and organic health conditions. Felitti and colleagues (1998) found that patients who experienced four or more types of adverse childhood events had higher rates of ischemic heart disease, cancer, stroke, chronic bronchitis, emphysema, diabetes, skeletal fractures, and hepatitis than their nontraumatized counterparts. The childhood events they studied included all types of maltreatment including psychological, physical, or contact sexual abuse and exposure to parental substance abuse, mental illness, intimate partner violence, or criminal behavior.

Since publication of that study, others have followed with similar findings. In the National Comorbidity Study, women who were maltreated as children had a ninefold increase in cardiovascular disease compared with nonmaltreated women (Batten, Aslan, Maciejewski, \& Mazure, 2004). In a sample of primary care 
patients, those who experienced childhood abuse or partner violence in adolescence or adulthood reported twice as many symptoms on a review of systems than their age-matched, nonabused counterparts. They were also more likely to abuse substances and report a wide variety of chronic pain syndromes (KendallTackett, Marshall, \& Ness, 2000, 2003).

Patients with diagnoses of posttraumatic stress disorder (PTSD) report similar symptoms. Data from the Canadian Community Health Survey $(N=36,984)$ indicated that participants with PTSD had significantly higher rates of cardiovascular disease, respiratory diseases, chronic pain syndromes, gastrointestinal illnesses, and cancer (Sareen, Cox, Stein, Afifi, Fleet, \& Asmundson, 2007). PTSD was also strongly associated with chronic fatigue syndrome and multiple-chemical sensitivity, but not diabetes.

PTSD following a human-made disaster showed similar health effects $(N=896$; Dirkzwager, van der Velden, Grievink, \& Yzermans, 2007). In this study, PTSD was associated with new vascular events as well as physician-reported vascular, musculoskeletal, and dermatological problems. These problems appeared even after controlling for previous health problem, smoking, and demographic characteristics.

Not surprisingly, people with PTSD use more health care services than those without PTSD. In a study of women seeking health care at Veterans' Administration facilities (Dobie et al., 2006), women with PTSD had more outpatient visits to the emergency department, primary care, medical or surgery subspecialities, ancillary services, and diagnostic tests. They had higher rates of hospitalizations and surgical procedures. Women with PTSD were significantly more likely to have a service-related disability, have chronic pain, and to be obese. They were also more likely to smoke and abuse alcohol. Seventy-five percent of the women with PTSD also screened positive for depression.

\section{Trauma and Chronic Pain}

Researchers studying chronic pain syndromes were among the first to note an association between trauma and health (Gross, Doerr, Caldirola, Guzinski, \& Ripley, 1980; HarropGriffiths et al., 1988). For example, Drossman,
Leserman, and colleagues (Drossman, Li, Leserman, Toomey, \& Hu, 1996; Leserman et al., 1996) found that $60 \%$ of women in treatment for functional gastrointestinal (GI) illness (such as irritable bowel syndrome) had a history of abuse, as children or adults. In another study (Drossman, Leserman, Li, Keefe, Hu, \& Toomey, 2000), half of the women referred to a GI treatment center had been physically or sexually abused. Fourteen percent reported severe abuse. Patients who had been more severely abused had poorer health status relative to the other patients.

Sachs-Ericsson, Kendall-Tackett, and Hernandez (2007), using data from the National Comorbidity Study, noted that patients with a history of childhood or partner abuse reported more pain when describing their current health symptoms. Van Houdenhove, Luyten, and Egle (2009) found that $64 \%$ of patients who had either fibromyalgia or chronic fatigue syndrome had experienced at least one type of either child or adult trauma. More concerning was that 39\% had experienced both, indicating a lifelong pattern of revictimization and abuse.

\section{Possible Mechanisms: The Psychoneuroimmunology of Trauma}

The negative health effects of trauma and PTSD, at this point, are fairly well established. A wide range of traumatic exposures and experiences can lead to poor health. Some of this is attributable directly to injury suffered during the trauma. Others result from the effects of the trauma. The question then becomes why does this occur? It's an important question to ask because it has implications for not only treating trauma survivors currently seeking care, but also for prevention of these health problems in the future.

Research in the field of PNI is a useful framework for understanding these effects. According to PNI research, many of these illnesses are because severe or overwhelming stress, and any resultant PTSD, alters and dysregulates the key systems that are part of the stress response. To understand these findings, it's helpful to review the three components of the stress system. These are described in the following section. 


\section{How Humans Respond to a Perceived Threat}

Human bodies have a number of interdependent mechanisms in place designed to preserve life in the face of danger: catecholamine, hypothalamic-pituitary-adrenal (HPA) axis, and immune response. In response to threat, the sympathetic nervous system responds by releasing the catecholamines norepinephrine, epinephrine, and dopamine. This is the fight-or-flight response. The HPA axis responds with a chemical cascade: the hypothalamus releases corticotrophin-releasing hormone $(\mathrm{CRH})$, which causes the pituitary to release adrenocorticotropin hormone (ACTH), which causes the adrenal cortex to release cortisol, a glucocorticoid.

There have been numerous studies over the past 15 years that have examined changes to the HPA axis and sympathetic nervous system that occur in the wake of traumatic events and/or PTSD (see Bremner, 2005; Kendall-Tackett, 2000, for reviews). Wilson, Friedman, and Lindy (2004) used McEwen's (1998) model of allostasis to explain the effects of PTSD on these systems and subsequent health problems. Allostasis refers to the body's attempt to maintain stability through stress and change. When the body is overwhelmed by stressors, allostatic load (or body "wear and tear") occurs. Allostatic load can threaten health if severe or chronic. Traumatic events can increase allostatic load by chronically activating the HPA axis and sympathetic nervous system. This dysregulation can be manifested with elevated or abnormally low levels of cortisol, and low or elevated levels of norepinephrine (KendallTackett, 2000). But the sympathetic nervous system and HPA axis are not the only systems involved.

The third part of this stress system is the immune response. The immune system responds to threat by releasing proinflammatory cytokines. These cytokines increase inflammation and serve the adaptive purpose of helping the body heal wounds and fight infection. Outside of the trauma field, there is a large and growing literature on the impact of these proinflammatory cytokines on health. A key finding of PNI research is that both physical and psychological stress can trigger the inflammatory response (Robles, Glaser, \& Kiecolt-Glaser, 2005). The cytokines studied most often are interleukin 1- $\beta$ (IL-1 $\beta$ ), interleukin-6 (IL-6), tumor necrosis factor- $\alpha$ (TNF- $\alpha$ ), and interferon- $\gamma$ (IFN- $\gamma$ ). These findings are an important part of the picture when studying the health effects of traumatic events and are the focus of this article.

The human stress response has a number of checks and balances built in to ensure that various components do not become overactive. Unfortunately, in the case of severe or overwhelming stress, the normal checks and balances fail, causing inflammation levels to be abnormally high. For example, cortisol (which is normally antiinflammatory and keeps proinflammatory cytokines in check) can actually change function under severe stress and potentiate the actions of IL-1 and IL-6 rather than inhibiting them (Dhabhar \& McEwen, 2001). When there are too many proinflammatory cytokines, or other inflammatory factors, humans become vulnerable to disease (McEwen, 2003).

\section{Inflammation in Trauma Survivors}

Although a relatively new area of study, several researchers using a psychoneuroimmunology framework have found that traumatic events increase levels of proinflammatory cytokines in trauma survivors. Elevated levels of proinflammatory cytokines or other inflammatory markers (e.g., C-reactive protein, fibrinogen) are associated with increased risk of a number of serious health problems including coronary heart disease, myocardial infarction, chronic pain syndromes, premature aging, impaired immune function, impaired wound healing, and Alzheimer's disease (Frasure-Smith \& Lesperance, 2005; Kiecolt-Glaser et al., 2005; Nivison, Guillozet-Bongaarts, \& Montine, in press; Pace, Hu, \& Miller, 2007; Surtees et al., 2008; Wilson et al., 2002).

Previous child maltreatment was related to high C-reactive protein levels when they were assessed 20 years later (Danese, Pariante, Caspi, Taylor, \& Poulton, 2007). The participants $(N=1,037)$ were part of the Dunedin Multidisciplinary Health and Development Study, a study of health behavior in a complete birth cohort in New Zealand. Researchers assessed the participants every two to three years throughout childhood, and every five to six years through age 32 . The impact of child maltreatment on inflammation was independent of 
other factors that could have accounted for the findings, such co-occurring life stresses in adulthood, early life risks, or adult health or health behavior. The effect was also doseresponsive; the more severe the abuse, the more severe the inflammation.

A similar finding resulted from a study of women abused by intimate partners. In this study, 62 women who had had abusive partners 8 to 11 years previously had significantly higher interferon-gamma (IFN- $\gamma$ ) levels than nonabused women (Woods et al., 2005). The women also had high rates of depression (52\%) and PTSD (39\%). Even several years after their abuse had ended, these women were still manifesting significant physical symptomatology. Inflammation was also found to be elevated in a study of 15 women who had been raped 24 to 72 hours after their assault, compared with 16 women who had not been sexually assaulted. Sexually assaulted women had higher ACTH, C-reactive protein, IL-6, IL-10, IFN- $\gamma$ than women in the nonassaulted group (Groër, Thomas, Evans, Helton, \& Weldon, 2006).

In a sample of 14 otherwise healthy patients with PTSD and a group of 14 patients without PTSD matched on age and gender of 14 patients without PTSD, von Kanel and colleagues (2006) investigated PTSD and blood coagulation by measuring clotting factors, fibrinogen, and D-dimer in the plasma. They found the more severe the PTSD, the higher the levels of fibrinogen and the clotting factor FVIII:C. They concluded that PTSD may elicit hypercoagulability, even at subthreshold levels, and this may increase risk for cardiovascular disease in trauma survivors. Fibrinogen is a soluble protein that aids in clotting. Because it increases the speed of platelet aggregation and thrombus formation, a high level of fibrinogen is a risk factor for cardiovascular disease.

\section{Emotional and Cognitive Factors}

The above-cited studies demonstrate that trauma and PTSD may directly alter the stressresponse systems of trauma survivors. While these findings are interesting, they do not fully explain the health effects of trauma. Additionally relevant is the fact that psychological factors, such as depression and hostility, can trigger the same inflammatory response. Furthermore, these effects can impair health even if the trauma survivor does not meet full criteria for PTSD.

\section{How Depression Influences Health}

Depression is one of the most common of all mood disorders and is a common symptom among trauma survivors. It also has a welldocumented negative impact on health-even beyond the effects of PTSD. In a large study of U.S. veterans $(N=35,715)$, depressed patients were at increased risk of dying over a 5-year period (Kinder et al., 2008). Patients with PTSD alone, or who had neither condition, did not have this increased risk.

Most of the research on depression and health has examined the link between depression and cardiovascular disease. Depression is a robust risk factor for cardiovascular disease, cardiovascular events, and cardiac-related mortality (Frasure-Smith \& Lesperance, 2005; Rutledge et al., 2006). Inflammation is a crucial factor in atherogenesis and the progression of coronary artery disease (Zouridakis, Avanzas, ArroyoEspliguero, Fredericks, \& Kaski, 2004). Inflammatory factors are not simply markers. They have a pathogenic role to play. And these inflammatory factors, including C-reactive protein and proinflammatory cytokines, are often elevated in depressed people.

Depression, inflammation, and heart disease. Several studies have found elevated C-reactive protein in depressed patients with heart disease. For example, in a study of men and women at risk for heart disease $(N=68)$, Taylor and colleagues (2006) found that depressed patients (mean age $=62$ ) had elevated C-reactive protein compared to matched controls. Surtees and colleagues (2008), in a sample of patients with ischemic heart disease, found that major depression in the previous year increased C-reactive protein levels. Those with higher C-reactive protein had an odds ratio of 2.02 for incident ischemic heart disease. This was true even when adjusting for other known cardiac risk factors including cigarette smoking, diabetes, systolic blood pressure, body mass index, and cholesterol.

Coaguability may also be a factor in depression's impact on heart disease. A study of patients 65 and older found that depression was related to both $\mathrm{C}$-reactive protein and coagulation factors (Kop et al., 2002). This study in- 
cluded 4,268 patients who were free of cardiovascular disease (mean age $=72.4$ years). Depressed men and women had elevated Creactive protein, elevated white blood cell count, and increased markers of coaguability. Similarly, coagulation was also related to depression and cardiovascular risk in a study of 3,292 perimenopausal women (Matthews et al., 2007). Over the five-year period, depressive symptoms were related to two indices of coagulation: fibrinogen and PAI-1.

Depression predicted cardiac symptoms in a sample of 750 women middle-aged women with suspected myocardial ischemia (Rutledge et al., 2006). The more severe the depression, the higher the mortality risk over the 2.3 year study period. Each point increase on the Beck Depression Inventory was associated with a $3 \%$ increase in mortality risk even after adjusting for age, disease severity, and other coronary artery disease risk factors.

Depression not only increases inflammation. It also increases the incidence of healthcompromising behavior (Kibler et al., in press). In a study of 254 patients with major mood disorders (Lu, Mueser, Rosenberg, \& Jankowski, 2008), researchers found that exposure to adverse childhood experiences was related to a number of health compromising factors. Those with increased exposure to ACEs had higher rates of risk-taking behaviors, diagnoses of substance use disorders, trauma in adulthood, PTSD, suicide attempts, medical service utilization (including younger age at first hospitalization), and homelessness. Thus, even within a population with severe mood disorders, adverse childhood experiences contribute to worse physical and mental health. Studies of other types of trauma have showed similar patterns (Kibler et al., in press).

\section{Hostility}

Depression is not the only cognitive factor that can increase risk of death and disease. Hostility has a similar effect. For people with a hostile world view, life is not benign. People high in trait hostility do not trust others, are suspicious and cynical about human nature, and tend to interpret the actions of others as aggressive (Smith, 1992). This psychological state also triggers the inflammatory response. Individuals who expect the worst from people be- come hypervigilant to rejection in social relationships, and this world view has discernable, physical sequelae (Kiecolt-Glaser \& Newton, 2001). In their review, Smith and Ruiz (2002) noted that people who are high in trait hostility are more prone to ischemia and constriction of the coronary arteries during mental stress. Trait hostility predicts new coronary events in previously healthy people. For patients who already have coronary heart disease, hostility speeds up the progression of the disease.

Unfortunately, hostility is common among trauma survivors. In a sample from primary care, $52 \%$ of female sexual abuse survivors indicated that they could not trust others compared with $17 \%$ of the nonabused women (Hulme, 2000). In a community sample, approximately half of sexual abuse survivors indicated that their views of themselves and others were very negative (Teegen, 1999). And in a sample of 90 women veterans (Butterfield, Forneris, Feldman, \& Beckham, 2000), women with PTSD reported significantly higher levels of hostility and had poorer overall health status than women without PTSD.

Like depression, hostility also triggers the inflammatory response system. In a study of 44 healthy, nonsmoking, premenopausal women, hostility increased levels of proinflammatory cytokines (IL- $1 \alpha$, IL- $1 \beta$, IL- 8 and TNF- $\alpha$; Suarez et al., 2004). The combination of depression and hostility was especially deleterious. The effect was dose-responsive: the more severe the depression and hostility, the greater the production of cytokines. A study with men had similar results (Suarez, 2003). Its author noted that increased levels of IL-6 predicted both future risk of cardiac events and all-cause mortality, and hypothesized that IL-6 may mediate the relationship between hostility and these health problems.

Kiecolt-Glaser et al. (2005) found that couples who were high in hostility had higher levels of circulating proinflammatory cytokines. As a result, the rate of wound healing for the highhostility couples was $60 \%$ slower than lowhostility couples. High-hostility couples had fewer cytokines at the wound site, where they were supposed to be, and high levels circulating systemically, where they were more likely to impair health and increase the risk of agerelated diseases. 
In his study of 135 healthy patients with no symptoms of diabetes, Suarez (2006) found that women with higher levels of depression and hostility had higher levels of fasting insulin, glucose, and insulin resistance. These findings did not hold true for men and they were independent of other risk factors for metabolic syndrome including BMI, age, fasting triglycerides, exercise regularity, or ethnicity. A study of 6,814 healthy men and women found that participants with higher levels of cynical distrust, chronic stress or depression had higher levels of C-reactive protein, IL-6, and fibrinogen (Ranjit et al., 2007)-all risk factors for heart disease.

\section{Sleep Disorders in PTSD}

Cognitive factors are not the only triggers for the inflammatory response system. Poor sleep is also associated with increased inflammation, including C-reactive protein, IL-6, IL-1, and TNF- $\alpha$ (Suarez \& Goforth, in press). Unfortunately, sleep disorders are also quite common among trauma survivors, especially those with PTSD, and could be another way that trauma impacts health.

A number of studies have documented disturbed sleep patterns in men and women who have experienced violence and resultant acute stress disorder (ASD) or PTSD. For example, in a European community sample, $68 \%$ of sexual abuse survivors reported having sleep difficulties, with $45 \%$ having repetitive nightmares (Teegen, 1999). In a French sample, 33\% of teens who had been raped reported poor quality sleep compared with $16 \%$ of the nonassaulted control group. Twenty-eight percent of the assaulted teens had nightmares (compared with $11 \%$ of nonassaulted teens), and $56 \%$ woke during the night (compared with 21\%; Choquet, Darves-Bornoz, Ledoux, Manfredi, \& Hassler, 1997).

Hulme (2000) found that sleep problems were common among women primary-care patients who were sexual abuse survivors. Fiftytwo percent of sexual abuse survivors reported that they could not sleep at night (compared with $24 \%$ of the nonabused group), and $36 \%$ reported nightmares (compared with 13\%). The most common symptom was intrusive thoughts, reported by $53 \%$ of sexual abuse survivors (compared with $18 \%$ of the nonabused group).
In a sample of battered women living in shelters $(N=50), 70 \%$ reported poor sleep quality, $28 \%$ went to bed very fatigued, and $40 \%$ woke up feeling very fatigued (Humphreys, Lee, Neylan, \& Marmar, 1999). Moreover, 82\% described one or more of the following characteristics of disturbed sleep: many wakings over the course of the night, restless sleep, and early morning waking. Six described vivid nightmares that included recent incidents of abuse.

In a study of sleep disorders in sexual assault survivors, $80 \%$ had either sleep-breathing or sleep-movement disorders. Both of these disorders were linked to higher levels of depression and suicidality. Women who had both types of sleep disorders had the most severe symptoms. For these women, fragmented sleep appeared to potentiate the symptoms they experienced after their assault, making coping with the aftermath even more difficult (Krakow, Artar, et al., 2000).

\section{How Sleep Impacts Health}

Sleep problems impact health in a number of negative ways. They increase the risk of coronary heart disease, Type- 2 diabetes, and hypertension. Sleep loss reduces lymphocyte count and the activity of the Natural Killer cells, making patients more vulnerable to infection. The link between sleep disturbances and inflammation appears to be bidirectional-with poor sleep increasing inflammation, and inflammation decreasing the quality of sleep (Suarez \& Goforth, in press).

The term "sleep disorders" cover a wide range of problems. Some of these disorders can be measured via patient questionnaire. Other sleep difficulties need to be measured via polysomnographic studies. These are necessary to detect common problems, such as sleepdisordered breathing (e.g., sleep apnea), and sleep-movement disorders (e.g., restless leg syndrome). Patients may not be aware of these problems, and therefore, would not be able to report them. Regardless of type of disorder, poor sleep quality has a profound effect on health. It compromises immune, metabolic, and neuroendocrine function, chronically activates the HPA axis, and even increases mortality risk (Carmichael \& Reis, 2005).

Sleep may also be compromised by quality of relationship with adult partner. This is of inter- 
est because several studies have found trauma survivors have higher rates of unstable relationships and are more likely to express dissatisfaction with their current partners than the general population (Kendall-Tackett, 2003). Two recent studies have examined the relationship between security of adult attachments and sleep quality. Sleep is a physiologically vulnerable state. In order to sleep soundly, people must feel sufficiently secure so they can downregulate vigilance and alertness. And to do so, one must be secure in social relationships (Troxel, Cyranowski, Hall, Frank, \& Buysee, 2007) or feel relatively safe.

In the first study, 78 married adults completed questionnaires about their sleep quality, quality of current partnership (secure vs. insecure attachment), and depression (Carmichael $\&$ Reis, 2005). The sleep questionnaire asked about seven aspects of sleep including perceived sleep quality, sleep latency (time to get to sleep), sleep duration, habitual sleep efficiency, sleep disturbances, and use of sleep medications. Using structural equation modeling, the researchers found that married participants who were anxious about their current relationships reported poorer sleep quality, even after controlling for depression. Women with insecure attachments were concerned that their partners were emotionally unavailable and not trustworthy. The researchers indicated that one limitation to their study was that they used a self-report measure of sleep, rather than assessing sleep directly.

Troxel and colleagues (2007) addressed that limitation by using polysomnographic studies to assess sleep directly. In a study of 107 women with recurrent major depression, marital status and security of that relationship predicted quality and efficiency of sleep. If women had anxious attachments, particularly if they were separated or widowed, they had a significantly smaller percentage of stage 3-4 sleep than women who were currently partnered and who had secure attachments. The authors noted the importance of stage 3-4 sleep in protecting individuals from cardiovascular and metabolic diseases.

McEwen (2003) reported that even short periods of sleep deprivation can elevate cortisol and glucose levels, and can increase both insulin and insulin resistance. Long-term sleep deprivation can seriously impair health, and this could be a health risk factor for women who are not in stable, secure relationships. The deleterious health effects of disturbed sleep have also been found in samples of trauma survivors. In a sample of female rape survivors with PTSD, trauma-related sleep disorders had an independent impact on health, even after controlling for both depression and PTSD (Clum, Nishith, \& Resick, 2001).

In summary, sleep disturbance is another common aftereffect of that trauma can negatively impact health. Unfortunately, sleep quality and disturbance are often not included in a normal trauma assessment, as they are also not in most other psychological assessments. But given its large impact on health, it should be assessed and treated before it can lead health problems.

\section{What Trauma Survivors Can Do to Improve Their Health}

Upon reviewing the literature on trauma and health, many practitioners (and some survivors) become concerned that nothing can be done to reverse these effects; that nothing can be done to halt their inevitable decline into poor health. Fortunately, that is not the case. In addition to standard treatments for PTSD, PNI research suggests some other interventions that may help counter some of the physiological changes that occur in the wake of traumatic events, in turn, lowering the risk of health problems.

\section{Downregulate the Inflammatory Response System}

Kiecolt-Glaser and colleagues (2007) noted that prior trauma "primes" the inflammatory response system so that there is heightened and more rapid rise in inflammation in response to stress. One key to improving the health of trauma survivors is downregulating this stress response and increasing resilience to stress. Research suggests two body-related adjuncts to traditional trauma treatment that specifically downregulate the inflammatory response system: Omega-3 fatty acids and exercise. These studies are summarized below. 


\section{Omega-3 Fatty Acids and the Stress Response}

Researchers have examined the impact of EPA and DHA, the long-chain omega-3 fatty acids, on stress. Several studies have suggested that EPA and DHA may have an adaptogenic role in stress by regulating and attenuating the stress response. For example, when college students had a high ratio of omega- $6 \mathrm{~s}$ to omega- $3 \mathrm{~s}$ (meaning low levels of omega-3s), they had more inflammation when exposed to a labinduced stressor. In contrast, students with higher levels of EPA/DHA had a lower inflammatory response to stress (Maes, Christophe, Bosmans, Lin, \& Neels, 2000). In other words, students were not deficient in EPA/DHA had an attenuated inflammatory response.

Similarly, Kiecolt-Glaser and colleagues (2007) found that EPA/DHA supplementation downregulated the stress response and its maladaptive impact on mood. In their study of 43 older adults, they noted that "even modest supplementation with n-3 PUFAs [Omega-3 polyunsaturated fatty acids] reduces plasma norepinephrine, an important link to the stress response" (p. 221). Patients who were both depressed and had low levels of omega-3s had the highest levels of proinflammatory cytokines. The authors noted that a diet that is low in EPA/DHA increases the risk of both depression and other diseases related to chronic inflammation.

EPA and DHA also specifically lower proinflammatory cytokines, which helps with depression and health problems associated with it. In a large population study $(N=1,123)$, high levels of EPA and DHA were related to lower levels of proinflammatory cytokines (IL- $1 \alpha$, IL-1 $\beta$, IL-6, and TNF- $\alpha$ ) and higher levels of anti-inflammatory cytokines, such as IL-10. For people with low levels of EPA and DHA, the opposite was true: they had high levels of proinflammatory cytokines and low levels of anti-inflammatory cytokines (Ferrucci et al., 2006). This study had several advantages over previous research. The sample in this study was representative of the population-not a specific subgroup. The fatty acids were directly measured in the plasma rather than estimated from patient dietary reports. Moreover, it was the first study that specifically examined the relationship between fatty acids and cytokines.
Some parallel work has been done with regard to Type 2 diabetes. In a review, Delarue and colleagues (2004) found that in healthy patients, fish oil reduced insulin resistance and plasma triglycerides and increased resilience to stress by decreasing the activity of the sympathetic nervous system. Fish oil did not reduce insulin resistance in patients with diabetes, but did lower triglycerides. The authors concluded that fish oil showed promise in the prevention of insulin resistance and related health problems. Given that trauma survivors also appear to be at increased risk of developing diabetes, the impact of EPA and DHA on insulin resistance could be another way that they positively impact health.

Sources of EPA and DHA. The chief dietary source of EPA and DHA is fish. According to population studies, people must eat 1 to 1.5 pounds of fish per week to achieve the mental health effects (Noaghiul \& Hibbeln, 2003). However, eating that much fish may not be safe because of contaminants in seafoodand not everyone likes fish. Fish oil is the best source of EPA and DHA in supplements. Fortunately, finding safe sources of fish oil is now relatively easy. The U.S. Pharmacoepia is an independent, not-for-profit organization that tests fish oil for contaminants and lists specific brands that they verify on their Web site (www.USP.org). The minimum dosage of DHA for possible prevention of depression is 200-400 $\mathrm{mg}$. The effective dosage for treatment of depression and other affective disorders is 1,000 mg of EPA. The FDA GRAS (generally recognized as safe) levels are $3000 \mathrm{mg}$ of EPA/DHA together, and $1500 \mathrm{mg}$ of DHA.

\section{Exercise and the Inflammatory Response}

Exercise is another intervention with stresslowering capabilities. Two clinical trials have found exercise as effective as sertraline (Zoloft) for the treatment of even major depression (Babyak et al., 2000; Blumenthal et al., 2007). Exercise also downregulates the inflammatory response system. Initially, exercise acts as an acute physical stressor and raises IL-6 and TNF- $\alpha$. An initial burst of these cytokines does not appear to be harmful. Indeed, and as Goebels, Mills, Irwin, and Ziegler (2000) point out, in a normally functioning immune system, high levels of cytokines trigger the body's anti- 
inflammatory mechanisms to keep inflammation in check.

Over a longer period of time, however, especially in people with chronically elevated proinflammatory cytokines, exercise lowers inflammation. Older adults, for example, are one group with higher levels of proinflammatory cytokines since levels naturally increase with aging. Because of this increased vulnerability in older adults, they are frequently the population of choice for studies on exercise, depression, and inflammation. The results of these studies are helpful for understanding the mechanism for exercise's impact on the stress response.

A study of adults ages 60 to 90 tested the effect of physical activity on perceived stress, mood, and quality of life. The researchers also assessed serum IL-6 and cortisol. The patients $(N=10)$ assigned to the exercise group were instructed to walk for 30 minutes, at a rate that would elevate their heart rate to $60 \%$ of its maximal capacity, five times a week for the 10 -week study. The control group was 10 older adults who were not engaging in physical activity. After the 10-week exercise intervention, the subjects had significantly lower stress on the Perceived Stress Scale, and improved mood and quality of life on the SF-36 Health Questionnaire. They reported better physical functioning, more vitality, better mental health, and less bodily pain. They also had a significant decrease in serum IL-6, an effect independent of an association between psychological variables and IL-6 (Starkweather, 2007).

Another study of older adults compared two types of workouts to see if either type lowered inflammation (Kohut et al., 2006). In this study, 83 adults, ages 64 to 87 , were randomized to either cardio or flexibility/resistance conditions. The cardio workouts were $45 \mathrm{~min}$ utes, at $60 \%$ to $80 \%$ of maximal cardiac effort, three times a week, for 10 months. The flexibility/resistance workouts were 45 minutes of resistance and flexibility training, three times a week, also for 10 months. Both types of exercise led to improved levels of depression, optimism, and sense of coherence, the three psychological measures the researchers assessed. At the end of 10 months, the cardio workout had the most impact on inflammation. Participants in the cardio condition had significant reductions in C-reactive protein, IL-6, and IL-18. TNF- $\alpha$ levels improved with both cardio and flexibility/resistance program. These effects were independent of depression, optimism or sense of coherence.

Exercise also had a positive effect on wound healing, and this is an indirect measure of systemic inflammation (Emery, Kiecolt-Glaser, Glaser, Malarky, \& Frid, 2006). In this study, participants were randomized into exercise and control conditions, and were then brought into the laboratory and given a punch biopsy. The researchers then monitored participants' rate of wound healing. The average number of days for the wound to heal in the exercise group was 29 days. In the control group, it was 38 days. Exercise one hour a day, three days a week, lowered perceived stress and improved wound healing. This study is of interest because we know from these researchers' other studies that wound healing is impaired when stress or hostility levels are high (e.g., Kiecolt-Glaser et al., 2005). Stress and hostility both increase systemic inflammation. When systemic inflammation is high, wound healing is impaired because proinflammatory cytokines are in the blood stream and not at the wound site where they belong. The Emery et al. (2006) study indicates that exercise improves wound healing by lowering levels of circulating systemic cytokines, and diverting cytokines to where they belong.

Overall level of fitness was also related to inflammation in another recent study (Hamer \& Steptoe, 2007). The sample consisted 207 men and women from London who had no history or symptoms of heart disease, and were not being treated for hypertension, inflammatory disease, or allergy. Participants were given one of two mentally stressful tasks in the laboratory (a computerized Stroop test or mirror tracing task). Researchers drew blood and measured heart rate via a submaximal exercise test. A high-systolic blood pressure indicated a low level of fitness. Participants who responded with higher systolic blood pressure to stress also had a higher IL-6 and TNF- $\alpha$ response. The TNF- $\alpha$ response to stress was five times greater in the low-fitness group compared to the high-fitness group. The authors concluded that participants who were physically fit had a lower inflammation response when under stress. They believed that this was another way that exercise protected individuals from heart disease and other conditions.

When starting an exercise program for trauma survivors, some cautions are in order. 
It's important to start this program slowly. Exercise that causes too rapid a rise in heart rate is similar to hypertension responses and may cause trauma survivors distress, including an increased PTSD response. Implementing an exercise program that builds up slowly allows patients to adapt to it psychologically and physically over time (C. Courtois, personal communication, January 3, 2009).

In summary, omega- $3 \mathrm{~s}$ and exercise both downregulate the stress response, particularly lowering levels of proinflammatory cytokines. Trauma and resultant PTSD can cause alterations in the stress response system that leave trauma survivors more vulnerable to subsequent life stress. These interventions give survivors the tools they need to lessen their heightened stress response and increase their resilience to stress. Further, chronically elevated inflammatory cytokines are related to an increased risk of disease. By lowering these through fish-oil supplements, exercise, or both, trauma survivors can also lessen their risk of disease.

\section{Treatment of Sleep Disorders}

As described earlier, sleep is often compromised in trauma survivors. This too increases their risk of health problems because disrupted sleep upregulates the stress response, including the level of proinflammatory cytokines (Suarez \& Goforth, in press). Krakow, Lowry, et al. (2000) noted that since sleep medicine is not well-integrated into trauma treatment, practitioners are often less effective than they could be if they also treated underlying sleep disorders.

To lessen the negative health effects of sleep problems, the first step is to assess trauma survivors for possible sleep disorders. Clinicians should ask about the onset of the sleep disorder. If clients have any psychiatric disorders, they should ask about the temporal sequence of when the sleep disorder and psychiatric disorders manifested. Did the symptoms of the psychiatric disorder predate the onset of sleep problems or vice versa? Polysomnigraphic studies can also reveal whether there are any sleepbreathing or sleep-movement disorders that might also be treated.
A brief overview of interventions for sleep difficulties. Interventions for sleep disorders include cognitive-behavioral approaches, assistive devices, and medications. Cognitivebehavioral approaches are the most common form of sleep treatments. In one recent review, it was effective for $70 \%$ to $80 \%$ of patients, and was comparable to sleep medications (Morin, 2004; Stepanski \& Perlis, 2000). Cognitivebehavioral interventions help with sleep because they produce changes in REM sleep. Cognitive approaches can also address worrying and rumination that may be at the base of primary or secondary insomnia (Morin \& Ware, 1996).

Cognitive therapy for insomnia includes three components: behavioral, cognitive, and educational, and can be most easily incorporated into standard trauma care. These include establishing regular bedtimes, not using the bed for anything but sleeping and sex, getting out of bed when unable to sleep, and eliminating naps during the day. There is also a relaxation component that focuses on both on autonomic relaxation techniques (e.g., progressive muscle relaxation), and cognitive techniques that stops the worrying that keeps people from sleeping. Finally, sleep-hygiene education helps people minimize behaviors that might interfere with sleep. This might include eliminating caffeine, exercise, upsetting reading or media, alcohol and smoking too close to bedtime (Morin, 2004; Stepanski \& Perlis, 2000). A combination of these approaches is effective for most patients with sleep disorders.

For sleep apnea, and other types of sleepbreathing disorders, a CPAP (Continuous Positive Airway Pressure) device can be helpful. This is a small unit includes a facial mask, which provides pressurized air to prevent the airway from collapsing while the patient sleeps. This ensures a better night's sleep. Some dental devices, such as a Mandibular Repositioning Device, can also help keep the airway open during sleep. A sleep-medicine specialist can help determine is a patient needs devices like these. The physician and therapist should be aware that devices such as these might be especially difficult for some trauma survivors because they have elements reminiscent of the trauma (the assault/domestic violence survivor who was choked, the sexual abuse survivor who was forced to perform oral sex and who felt smothered during the experience). They should, 
therefore, work carefully with the survivor to implement the use of these devices, or in some cases, may need to find alternative approaches that are easier to tolerate (C. Courtois, personal communication, January 3, 2009).

Although medications are not always part of trauma treatment, for patients with sleep disorders, the Serotonin-2 Antagonists/Reuptake Inhibitors (SARIs), such as trazadone (Desyrel) and nefazadone (Serzone), can be helpful adjuncts. When they are administered with a Selective Serotonin Reuptake Inhibitor (SSRI), SARIs boost the actions of these drugs, and reverses medication-induced insomnia. Trazadone suppresses REM sleep, which can reduce the number of nightmares patients experience (Lange et al., 1999). In contrast, nefazadone increases REM sleep and improves overall sleep quality (Bezchilibnzyk-Butler \& Jeffries, 1999). Both of these drugs may be too sedating for some patients, however (Friedman, 2001). For a more detailed discussion of medications for PTSD, see Friedman (2004).

\section{Conclusion}

Until recently in the trauma field, researchers and clinicians have tended to focus predominantly on mental health sequelae, largely leaving physical health sequelae for other health care providers to deal with. This oversight in the field has been much more recognized in recent years and the physical/medical effects associated with trauma and its aftermath are now well researched and well recognized (e.g., Schnurr \& Green, 2003). For more than 20 years now, PNI researchers have demonstrated that what individuals think and feel has a measurable effect on the immune system and response to stress, demonstrating that the line between physical and mental health is not as solid as we once believed. Indeed, mental health sequelae, such as depression, are both outcomes and mechanisms that lead to poor health. One of the more hopeful findings of this research is that these trauma-related effects can be managed and possibly reversed. Trauma survivors can take a number of specific actions to downregulate their stress response and halt their decline into serious health problems. For trauma survivors working to regain control of their lives, that is good news indeed.

\section{References}

Babyak, M., Blumenthal, J. A., Herman, S., Khatri, P., Doraiswamy, M., Moore, K., et al. (2000). Exercise treatment for major depression: Maintenance of therapeutic benefit at 10 months. Psychosomatic Medicine, 62, 633-638.

Batten, S. V., Aslan, M., Maciejewski, P. K., \& Mazure, C. M. (2004). Childhood maltreatment as a risk factor for adult cardiovascular disease and depression. Journal of Clinical Psychiatry, 65, 249-254.

Bezchilibnzyk-Butler, K. Z., \& Jeffries, J. J. (1999). Clinical handbook of psychotropic drugs (9th ed.). Seattle, WA: Hogrefe \& Huber.

Blumenthal, J. A., Babyak, M. A., Doraiswamy, P. M., Watkins, L., Hoffman, B. M., Barbour, K. A., et al. (2007). Exercise and pharmacotherapy in the treatment of major depressive disorder. Psychosomatic Medicine, 69, 587-596.

Bremner, J. D. (2005). The neurobiology of childhood sexual abuse in women with posttraumatic stress disorder. In K. A. Kendall-Tackett (Ed.), Handbook of women, stress and trauma (pp. 181203). New York: Taylor \& Francis.

Butterfield, M. I., Forneris, C. A., Feldman, M. E., \& Beckham, J. C. (2000). Hostility and functional health status in women veterans with and without posttraumatic stress disorder: A preliminary study. Journal of Traumatic Stress, 13, 735-741.

Carmichael, C. L., \& Reis, H. T. (2005). Attachment, sleep quality, and depressed affect. Health Psychology, 24, 526-531.

Choquet, M., Darves-Bornoz, J-M., Ledoux, S., Manfredi, R., \& Hassler, C. (1997). Self-reported health and behavioral problems among adolescent victims of rape in France: Results of a crosssectional survey. Child Abuse and Neglect, 21, 823-832.

Clum, G. A., Nishith, P., \& Resick, P. A. (2001). Trauma-related sleep disturbance and self-reported physical health symptoms in treatment-seeking female rape victims. Journal of Nervous \& Mental Disease, 189, 618-622.

Danese, A., Pariante, C. M., Caspi, A., Taylor, A., \& Poulton, R. (2007). Childhood maltreatment predicts adult inflammation in a life-course study. Proceedings of the National Academy of Sciences USA, 104, 1319-1324.

Delarue, J., LeFoll, C., Corporeau, C., \& Lucas, D. (2004). N-3 long-chain polyunsaturated fatty acids: A nutritional tool to prevent insulin resistance associated to type 2 diabetes and obesity? Reproductive Nutrition \& Development, 44, 289-299.

Dhabhar, F. D., \& McEwen, B. S. (2001). Bidirectional effects of stress and glucocorticoid hormones on immune function: Possible explanations for paradoxical observations. In R. Ader, D. L. 
Felten, \& N. Cohen (Eds.), Psychoneuroimmunology (3rd ed., Vol. 1, pp. 301-338). New York: Academic Press.

Dirkzwager, A. J., van der Velden, P. G., Grievink, L., \& Yzermans, C. J. (2007). Disaster-related posttraumatic stress disorder and physical health. Psychosomatic Medicine, 69, 435-440.

Dobie, D. J., Maynard, C., Kivlahan, D. R., Johnson, K. M., Simpson, T., David, A. C., et al. (2006). Posttraumatic stress disorder screening status is associated with increased VA medical and surgical utilization in women. Journal of General Internal Medicine, 21, S58-64.

Drossman, D. A., Leserman, J., Li, Z., Keefe, F., Hu, Y. J. B., \& Toomey, T. C. (2000). Effects of coping on health outcome among women with gastrointestinal disorders. Psychosomatic Medicine, 62, 309-317.

Drossman, D. A., Li, Z., Leserman, J., Toomey, T. C., \& Hu, Y. J. B. (1996). Health status by gastrointestinal diagnosis and abuse history. Gastroenterology, 110, 999-1007.

Emery, C. F., Kiecolt-Glaser, J. K., Glaser, R., Malarky, W. B., \& Frid, D. J. (2006). Exercise accelerates wound healing among health older adults: A preliminary investigation. The Journals of Gerontology Series A, Biological Sciences and Medical Sciences, 60, 1432-1436.

Felitti, V. J., Anda, R. F., Nordenberg, D., Williamson, D. F., Spitz, A. M., Edwards, V. J., et al. (1998). Relationship of childhood abuse and household dysfunction to many of the leading causes of death in adults. American Journal of Preventive Medicine, 14, 245-258.

Ferrucci, L., Cherubini, A., Bandinelli, S., Bartali, B., Corsi, A., Lauretani, F., et al. (2006). Relationship of plasma polyunsaturated fatty acids to circulating inflammatory markers. Journal of Clinical Endocrinology \& Metabolism: Clinical and Experimental, 91, 439-446.

Frasure-Smith, N., \& Lesperance, F. (2005). Reflections on depression as a cardiac risk factor. Psychosomatic Medicine, 67, S19-S25.

Friedman, M. J. (2001). Posttraumatic stress disorder: The latest assessment and treatment strategies. Kansas City, MO: Compact Clinicals.

Friedman, M. J. (2004). Allostatic vs. empirical perspectives on pharmacotherapy for PTSD. In J. P. Wilson, M. J. Friedman, \& J. D. Lindy (Eds.), Treating psychological trauma and PTSD (pp. 94124). New York: Guilford Press.

Goebel, M. U., Mills, P. J., Irwin, M. R., \& Ziegler, M. G. (2000). Interleukin-6 and tumor necrosis factor-alpha production after acute psychological stress, exercise, and infused isoproterenol: Differential effects and pathways. Psychosomatic Research, 62, 591-598.
Groër, M. W., Thomas, S. P., Evans, G. W., Helton, S., \& Weldon, A. (2006). Inflammatory effects and immune system correlates of rape. Violence and Victims, 21, 796-808.

Gross, R. J., Doerr, H., Caldirola, D., Guzinski, G. M., \& Ripley, H. S. (1980). Borderline syndrome and incest in chronic pelvic pain patients. International Journal of Psychiatry in Medicine, 10, 79-96.

Hamer, M., \& Steptoe, A. (2007). Association between physical fitness, parasympathetic control, and proinflammatory responses to mental stress. Psychosomatic Medicine, 69, 660-666.

Harrop-Griffiths, J., Katon, W., Walker, E., Holm, L., Russo, J., \& Hickok, L. (1988). The association between chronic pelvic pain, psychiatric diagnoses, and childhood sexual abuse. Obstetrics and Gynecology, 71, 589-593.

Hulme, P. A. (2000). Symptomatology and health care utilization of women primary care patients who experienced childhood sexual abuse. Child Abuse and Neglect, 24, 1471-1484.

Humphreys, J. C., Lee, K. A., Neylan, T. C., \& Marmar, C. R. (1999). Sleep patterns of sheltered battered women. Journal of Nursing Scholarship, 31, 139-143.

Kendall-Tackett, K. A. (2000). Physiological correlates of childhood abuse: Chronic hyperarousal in PTSD, depression, and irritable bowel syndrome. Invited review. Child Abuse \& Neglect, 24, 799810 .

Kendall-Tackett, K. A. (2003). Treating the lifetime health effects of childhood victimization. Kingston, NJ: Civic Research Institute.

Kendall-Tackett, K. A. (2007). Cardiovascular disease and metabolic syndrome as sequelae of violence against women: A psychoneuroimmunology approach. Trauma, Violence and Abuse, 8, 117126.

Kendall-Tackett, K. A., Marshall, R., \& Ness, K. E. (2000). Victimization, healthcare use, and health maintenance. Family Violence \& Sexual Assault Bulletin, 16, 18-21.

Kendall-Tackett, K. A., Marshall, R., \& Ness, K. E. (2003). Chronic pain syndromes and violence against women. Women and Therapy, 26, 45-56.

Kibler, J. L., Joshi, K., \& Hughes, E. E. (in press). Cognitive and behavioral reactions to stress among adults with PTSD: Implications for immunity and health. In The psychoneuroimmunology of chronic disease: Exploring the links between inflammation, stress, and illness. Washington, DC: American Psychological Association.

Kiecolt-Glaser, J. K., Belury, M. A., Porter, K., Beversdoft, D., Lemeshow, S., \& Glaser, R. (2007). Depressive symptoms, omega-6: Omega-3 fatty acids, and inflammation in older adults. Psychosomatic Medicine, 69, 217-224. 
Kiecolt-Glaser, J. K., Loving, T. J., Stowell, J. R., Malarky, W. B., Lemeshow, S., Dickinson, S. L., et al. (2005). Hostile marital interactions, proinflammatory cytokine production, and wound healing. Archives of General Psychiatry, 62, 13771384.

Kiecolt-Glaser, J. K., \& Newton, T. L. (2001). Marriage and health: His and hers. Psychological Bulletin, 127, 472-503.

Kinder, L. S., Bradley, K. A., Katon, W. J., Ludman, E., McDonell, M. B., \& Bryson, C. L. (2008). Depression, posttraumatic stress disorder, and mortality. Psychosomatic Medicine, 70, 20-26.

Kohut, M. L., McCann, D. A., Konopka, D. W. R., Cunnick, J. E., Franke, W. D., Castillo, M. C., et al. (2006). Aerobic exercise, but not flexibility/ resistance exercise, reduces serum IL-18, CRP, and IL-6 independent of $\beta$-blockers, BMI, and psychosocial factors in older adults. Brain, Behavior, \& Immunity, 20, 201-209.

Kop, W. J., Gottdiener, J. S., Tangen, C. M., Fried, L. P., McBurnie, M. A., Walston, J., et al. (2002). Inflammation and coagulation factors in persons $>65$ years of age with symptoms of depression but without evidence of myocardial ischemia. American Journal of Cardiology, 89, 419-424.

Krakow, B., Artar, A., Warner, T. D., Melendez, D., Johnston, L., Hollifield, M., et al. (2000). Sleep disorder, depression, and suicidality in female sexual assault survivors. Crisis, 21, 163-170.

Krakow, B., Lowry, C., Germain, A., Gaddy, L., Hollifield, M., Koss, M., et al. (2000). A retrospective study on improvements in nightmares and post-traumatic stress disorder following treatment for co-morbid sleep-disordered breathing. Journal of Psychosomatic Research, 49, 291-298.

Lange, A., deBeurs, E., Dolan, C., Lachnit, T., Sjollema, S., \& Hanewald, G. (1999). Long-term effects of childhood sexual abuse: Objective and subjective characteristics of the abuse and psychopathology in later life. Journal of Nervous and Mental Disease, 187, 150-158.

Leserman, J., Drossman, D. A., Li, Z., Toomey, T. C., Nachman, G., \& Glogau, L. (1996). Sexual and physical abuse history in gastroenterology practice: How types of abuse impact health status. Psychosomatic Medicine, 58, 4-15.

Lu, W., Mueser, K. T., Rosenberg, S. D., \& Jankowski, M. K. (2008). Correlates of adverse childhood experiences among adults with severe mood disorders. Psychiatric Services, 59, $1018-$ 1026.

Maes, M., Christophe, A., Bosmans, E., Lin, A., \& Neels, H. (2000). In humans, serum polyunsaturated fatty acid levels predict the response of proinflammatory cytokines to psychologic stress. Biological Psychiatry, 47, 910-920.
Matthews, K. A., Schott, L. L., Bromberger, J., Cyranowski, J., Everson-Rose, S. A., \& Sowers, M. F. (2007). Associations between depressive symptoms and inflammatory/hemostatic markers in women during the menopausal transition. Psychosomatic Medicine, 69, 124-130.

McEwen, B. S. (1998). Protective and damaging effects of stress mediators: Allostasis and allostatic load. New England Journal of Medicine, 338, 171179.

McEwen, B. S. (2003). Mood disorders and allostatic load. Biological Psychiatry, 54, 200-207.

Morin, C. M. (2004). Cognitive-behavioral approaches to the treatment of insomnia. Journal of Clinical Psychiatry, 65, 33-40.

Morin, C. M., \& Ware, J. C. (1996). Sleep and psychopathology. Applied and Preventive Psychology, 5, 211-224.

Nivison, M., Guillozet-Bongaarts, A. L., \& Montine, T. J. (in press). Inflammation, fatty acid oxidation and neurodegenerative disease. In K. A. KendallTackett (Ed.), The psychoneuroimmunology of chronic disease: Exploring the links between inflammation, stress, and illness. Washington, DC: American Psychological Association.

Noaghiul, S., \& Hibbeln, J. R. (2003). Cross-national comparisons of seafood consumption and rates of bipolar disorders. American Journal of Psychiatry, 160, 2222-2227.

Pace, T. W., Hu, F., \& Miller, A. H. (2007). Cytokine-effects on glucocorticoid receptor function: Relevance to glucocorticoid resistance and the pathophysiology and treatment of major depression. Brain, Behavior and Immunity, 21, 9-19.

Ranjit, N., Diez-Roux, A. V., Shea, S., Cushman, M., Seeman, T., Jackson, S. A., et al. (2007). Psychosocial factors and inflammation in the multi-ethnic study of atherosclerosis. Archives of Internal Medicine, 167, 174-181.

Robles, T. F., Glaser, R., \& Kiecolt-Glaser, J. K. (2005). Out of balance: A new look at chronic stress, depression, and immunity. Current Directions in Psychological Science, 14, 111-115.

Rutledge, T., Reis, S. E., Olson, M., Owens, J., Kelsey, S. F., Pepine, C. J., et al. (2006). Depression is associated with cardiac symptoms, mortality risk, and hospitalization among women with suspected coronary disease: The NHLBI-sponsored WISE study. Psychosomatic Medicine, 68, 217-223.

Sachs-Ericsson, N., Kendall-Tackett, K. A., \& Hernandez, A. (2007). Childhood abuse and pain in the National Comorbidity Study. Child Abuse \& Neglect, 31, 531-547.

Sareen, J., Cox, B. J., Stein, M. B., Afifi, T. O., Fleet, C., \& Asmundson, G. J. (2007). Physical and mental comorbidity, disability, and suicidal behavior associated with posttraumatic stress disorder in a 
large community sample. Psychosomatic Medicine, 69, 242-248.

Schnurr, P. P., \& Green, B. L. (2003). Trauma and health: Physical health consequences of exposure to extreme stress. Washington, DC: American Psychological Association.

Smith, T. W. (1992). Hostility and health: Current status of a psychosomatic hypothesis. Health Psychology, 11, 139-150.

Smith, T. W., \& Ruiz, J. M. (2002). Psychosocial influences on the development and course of coronary heart disease: Current status and implications for research and practice. Journal of Consulting and Clinical Psychology, 70, 548-568.

Starkweather, A. R. (2007). The effects of exercise on perceived stress and IL-6 levels among older adults. Biological Nursing Research, 8, 1-9.

Stepanski, E. J., \& Perlis, M. L. (2000). Behavioral sleep medicine: An emerging subspecialty in health psychology and sleep medicine. Journal of Psychosomatic Research, 49, 343-347.

Suarez, E. C. (2003). Joint effect of hostility and severity of depressive symptoms on plasma Interleukin-6 concentration. Psychosomatic Medicine, 65, 523-527.

Suarez, E. C. (2006). Sex differences in the relation of depressive symptoms, hostility, and anger expression to indices of glucose metabolism in nondiabetic adults. Health Psychology, 25, 484-492.

Suarez, E. C., \& Goforth, H. (in press). Sleep and inflammation: A potential link to chronic diseases. In K. A. Kendall-Tackett (Ed.), The psychoneuroimmunology of chronic disease: Exploring the links between inflammation, stress, and illness. Washington, DC: American Psychological Association.

Suarez, E. C., Lewis, J. G., Krishnan, R. R., \& Young, K. H. (2004). Enhanced expression of cytokines and chemokines by blood monocytes to in vitro lipopolysaccharide stimulation are associated with hostility and severity of depressive symptoms in healthy women. Psychoneuroendocrinology, 29, $1119-1128$.

Surtees, P. G., Wainwright, N. W. J., Bockholdt, S. M., Luben, R. N., Warcham, N. J., \& Khaw, K.-T. (2008). Major depression, C-reactive protein, and incident ischemic heart disease in health men and women. Psychosomatic Medicine, 70, $850-855$.

Taylor, C. B., Conrad, A., Wilhelm, F. H., Neri, E., DeLorenzo, A., Kramer, M. A., et al. (2006). Psy- chophysiological and cortisol responses to psychological stress in depressed and nondepressed older men and women with elevated cardiovascular disease risk. Psychosomatic Medicine, 68, 538-546.

Teegen, F. (1999). Childhood sexual abuse and longterm sequelae. In A. Maercker, M. Schutzwohl, \& Z. Solomon (Eds.), Posttraumatic stress disorder: A lifespan developmental perspective (pp. 97112). Seattle: Hogrefe \& Huber.

Troxel, W. M., Cyranowski, J. M., Hall, M., Frank, E., \& Buysee, D. J. (2007). Attachment anxiety, relationship context, and sleep in women with recurrent major depression. Psychosomatic Medicine, 69, 692-699.

Van Houdenhove, B., Luyten, P., \& Egle, U. T. (2009). The role of childhood trauma in chronic pain and fatigue. In V. L. Banyard, V. J. Edwards, \& K. A. Kendall-Tackett (Eds.) Trauma and physical health: Understanding the effects of extreme stress and of psychological harm. London: Routledge.

von Kanel, R., Hepp, U., Buddeberg, C., Keel, M., Mica, L., Aschbacher, K., et al. (2006). Altered blood coagulation in patients with posttraumatic stress disorder. Psychosomatic Medicine, 68, 598 604.

Wilson, C. J., Finch, C. E., \& Cohen, H. J. (2002). Cytokines and cognition: The case for a head-totoe inflammatory paradigm. Journal of the American Geriatrics Society, 50, 2041-2056.

Wilson, J. P., Friedman, M. J., \& Lindy, J. D. (2004). Treatment goals for PTSD. In J. P. Wilson, M. J. Friedman, \& J. D. Lindy (Eds.), Treating psychological trauma and PTSD (pp. 3-27). New York: Guilford Press.

Woods, A. B., Page, G. G., O'Campo, P., Pugh, L. C., Ford, D., \& Campbell, J. C. (2005). The mediation effect of posttraumatic stress disorder symptoms on the relationship of intimate partner violence and IFN-gamma levels. American Journal of Community Psychology, 36, 159-175.

Zouridakis, E., Avanzas, P., Arroyo-Espliguero, R., Fredericks, S., \& Kaski, J. C. (2004). Markers of inflammation and rapid coronary artery disease progression in patients with stable angina pectoris. Circulation, 110, 1747-1753.

Received January 1, 2009

Revision received January 8, 2009 Accepted January 8, 2009 